\title{
Precise genome editing without exogenous donor DNA via retron editing system in human cells
}

\section{Dear Editor,}

CRISPR-Cas9 mediated seamless genome editing can be achieved by incorporating donor DNA into the CRISPR-Cas9 target loci via homology-directed repair (HDR), albeit with relative low efficiency due to the inefficient delivery of exogenous DNA (Cox et al., 2015; Gao, 2021). Retrons are bacterial phage-defense related operons composed of a specialized reverse transcriptase (RT) and a relevant noncoding RNA (ncRNA) which can be partially reverse transcribed by RT initiating at a conserved guanosine (G) residue to produce a multicopy single-stranded DNA (msDNA) (Yee et al., 1984; Millman et al., 2020). After being reverse transcribed, the msDNA is usually covalently tethered to the ncRNA through the $2^{\prime}, 5^{\prime}$-phosphodiester bond between the priming $G$ in ncRNA and $5^{\prime}$ end of msDNA (Dhundale et al., 1987). The reverse transcription process, of which the specialized $R T$ recognizes the unique secondary structure of retron ncRNA, is highly specific (Hsu et al., 1989). Additionally, desired msDNA can be generated in vivo by replacing the dispensable region of retron ncRNA with desired sequences (Mirochnitchenko et al., 1994; Simon et al., 2019). Therefore, retrons are promising biological sources for in vivo generation of DNA donors for HDR-mediated precise genome editing.

Recently, several studies have shown that retrons coupled with CRISPR-Cas9 could enhance precise genome editing via $H D R$ in bacterium and yeast through fusing guide RNA (gRNA) to the $3^{\prime}$ end of retron ncRNA, producing multicopy single-stranded DNA (msDNA) covalently tethered to gRNA (Sharon et al., 2018; Lim et al., 2020; Schubert et al., 2021). In this study, we further engineered retrons by fusing Cas9 with $E$. coli RT from different clades and joining gRNA at the $5^{\prime}$ end of retron ncRNA, and found that retron editing can achieve precise genome editing efficiently in human cells. By co-expression of Cas9-RT fusions and retron-ncRNA gRNA (rgRNA) in HEK293T cells, we demonstrated the rate of retron editing-mediated HDR events at endogenous genomic loci was up to $10 \%$. We expect our retron editing system could aid in advancing the ex vivo and in vivo therapeutic applications of retrons.
Given that HDR-mediated precise editing can be enhanced by increasing the local abundance of donor DNA, and CRISPEY strategy showed highly efficient editing in yeast genome by in situ expressing retron-gRNA chimeric molecule (Sharon et al., 2018). Therefore, we attempted to in situ reverse transcribe the donor msDNA covalently tethered to gRNA by fusing retron ncRNA at the $5^{\prime}$ or $3^{\prime}$ end of gRNA, termed as the retron-ncRNA gRNA (rgRNA) (Fig. 1A and $1 \mathrm{~B})$. Four experimentally validated $E$. coli retrons were evaluated for in vivo production of msDNA in human cells (Figs. 1B and S1). Additionally, we fused retron RT to the amino terminus or carboxy terminus of Cas9 with XTEN linker to increase the spatial proximity between RT and Cas9, which may enhance the retron editing in human cells by increasing the abundance of donor msDNA in the vicinity of DSB stimulated by Cas9 (Fig. 1B).

We first sought to test the relative abundance of msDNA in human cells. We found all of four selected RTs enabled the expression of msDNA in human cells, and retron RT combining with $3^{\prime}$ rgRNA showed higher expression of msDNA comparing to that with $5^{\prime}$ rgRNA (Fig. 1C). The results indicated that retron RT were reverse transcription-functional in human cells, inspiring us to further study the potential of retron editing at human endogenous genomic loci.

Next, we investigated the potential of retron editing in human HEK293T cells (Figs. 2 and S2). We transfected the HEK293T cells with plasmids expressing different Cas9-RT fusions targeting EMX1 locus and HEK3 site (Anzalone et al., 2019), and plasmids expressing $5^{\prime}$ rgRNA or $3^{\prime}$ rgRNA that can produce msDNA with 120 nucleotide (nt)-long homology donor sequences as well (Fig. $2 \mathrm{~A}$ and $2 \mathrm{~B}$ ). To determine the retron editing-mediated HDR efficiency at $E M X 1$ and $H E K 3$, we performed deep sequencing. Among four retrons tested, Ec86, Ec73 and Ec107 achieved varying degrees of precise editing and Ec73 combining with CRISPR-Cas9 showed highest activity, up to $10 \%$ (Fig. 2C). Restriction-fragment length polymorphism (RFLP), confirmed the deep sequencing results (Fig. S2A). In addition, retron editing with shortened homology donor sequences $(90$ nt) also enabled precise editing, including insertions and transversions (Fig. S2B). To test that retron editing is RT 
A

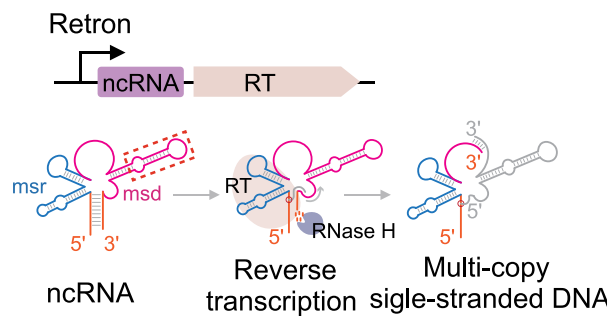

B RT-Cas9

RT XTEN Cas 9

$\mathrm{RT}=\mathrm{Ec} 48 \mathrm{RT}, \mathrm{Ec73} \mathrm{RT}, \mathrm{Ec86} \mathrm{RT}$ or Ec107 RT

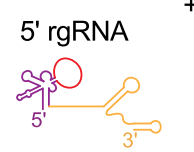

Retron ncRNA with HEK3 donor

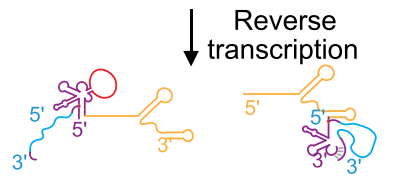

Donor msDNA is covalently tethered to gRNA

by $2^{\prime}, 5^{\prime}$-phosphodiester bond at the $5^{\prime}$ end

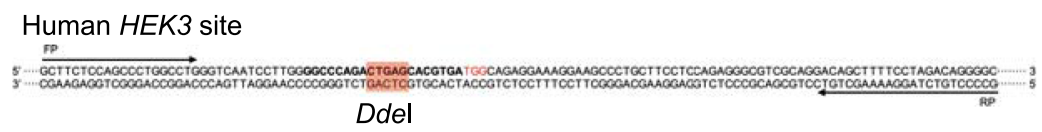

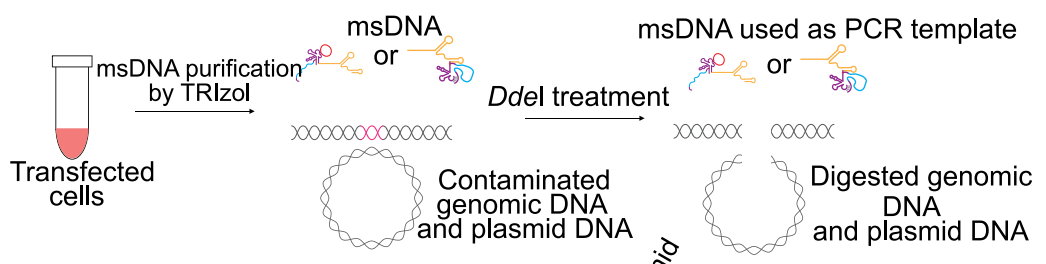

$\begin{array}{llllllllllll}2 & 3 & 4 & 5 & 6 & 7 & 8 & 9 & 10 & 11 & 12 & Q^{2}\end{array}$

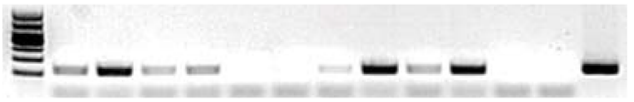

1: Ec86 RT-Cas9 + 5'rgRNA 7: Ec73 RT-Cas9 + 5'rgRNA

2: Ec86 RT-Cas9 + 3'rgRNA 8: Ec73 RT-Cas9 + 3'rgRNA

3: Ec48 RT-Cas9 + 5'rgRNA 9: Ec107 RT-Cas9 + 5'rgRNA

4: Ec48 RT-Cas9 + 3'rgRNA 10: Ec107 RT-Cas9 + 3'rgRNA

5: Cas9 + 5' 73 rgRNA 11: Cas9 + 5' Ec107 rgRNA

6: Cas 9 + 3' 73 rgRNA 12: Cas9 + 3' Ec107 rgRNA

Figure 1. Retron-mediated generation of msDNA in human cells. (A) Schematic representing retron-mediated msDNA production. Retrons are composed of non-coding RNA (ncRNA) and a specific reverse transcriptase (RT). The msr and msd region of retron ncRNA form secondary structure which can be specifically recognized by relevant retron RT reverse transcribing part of msd region, generating multicopy single-stranded DNA (msDNA). After reverse trancription, the msd template is degraded by RNase $\mathrm{H}$. The replaceable region of ncRNA, in which the donor sequence is inserted, is marked by red-dotted box. Red circle indicates $2^{\prime}, 5^{\prime}-$ phosphodiester bond between $2^{\prime}$ end of priming guanosine $(\mathrm{G})$ and $5^{\prime}$ end of the msDNA. (B) Plasmids construction strategies for retron-mediated generation of msDNA in human cells. Human codon-optimized RT-XTEN-spCas9 fusions are driven by the CAG promoter, whereas the $5^{\prime}$ extended retron-ncRNA gRNA ( $5^{\prime}$ rgRNA), in which the retron ncRNA is fused to the $5^{\prime}$ of gRNA, or $3^{\prime}$ rgRNA, is driven by the EF1 $\alpha$ promoter. Four E. coli retrons (Ec48 RT, Ec73 RT, Ec86 RT and Ec107 RT) from different clades were used in our retron editing system. The replaceable region of retron ncRNA was replaced by a 122 nt modified HEK3 sequencce. (C) Determination of msDNA level. The msDNA abundance was determined by PCR. PCR was conducted using the Ddel digested product as template.

activity-dependent, point mutations of D189A and D190A were introduced to the predicted active site of the Ec73 (Figs. 2D and S3), generating a catalytically dead Ec73 (dEc73). As expected, compared with Cas9 fused with wildtype Ec73, Cas9 fused with dEc73 or Cas9 only dramatically reduced the retron editing-mediated HDR efficiency (Fig. 2D). Together, our results indicated that Cas9-Ec73 RT fusion combining with $3^{\prime}$ Ec73 rgRNA can be harnessed for efficient precise genome editing in human cells (Fig. 2E). Of note, besides HDR events, we detected relatively high frequency of indels simulated by retron editing system (Figs. S2 and S4), suggesting Cas9-RT fusions co-expressing with relevant rgRNA retained the double stranded DNA cleavage activity of Cas9.

In addition, four of reported CRISPR-Cas9 off-target sites relative to EMX1 locus were checked to determine the offtargeting effects of retron editing (Fig. S5). Although, Cas9Ec73RT with rgRNA induced similar rate of indels at on- target sites as Cas9 with sgRNA (Fig. S5B), Cas9-Ec73RT with rgRNA showed reduced activity at the four known offtarget sites, with 4.9-fold lower average frequency than that of Cas9 with sgRNA. To be noted, the off-targeting effects of msDNA should be checked before further application of retron editing, although the frequency of random insertion of ssDNA is much lower than that of dsDNA.

The ability to write any modification of interest into the genome is a long-sought goal of biotechnology. Retrons, capable of producing intracellular SsDNA as donor with high specificity, are promising biological sources for precise genome editing. In this study, we demonstrate that different retrons are functional in human cells. Moreover, by co-expressing Cas9-RT fusions and $3^{\prime}$ extended retron-ncRNA gRNA, retron editing could mediate precise genome editing in human cell. Compared with ssDNA-mediated HDR in 293T cells, no exogenous ssDNA donor is required in retron editing system. 
A

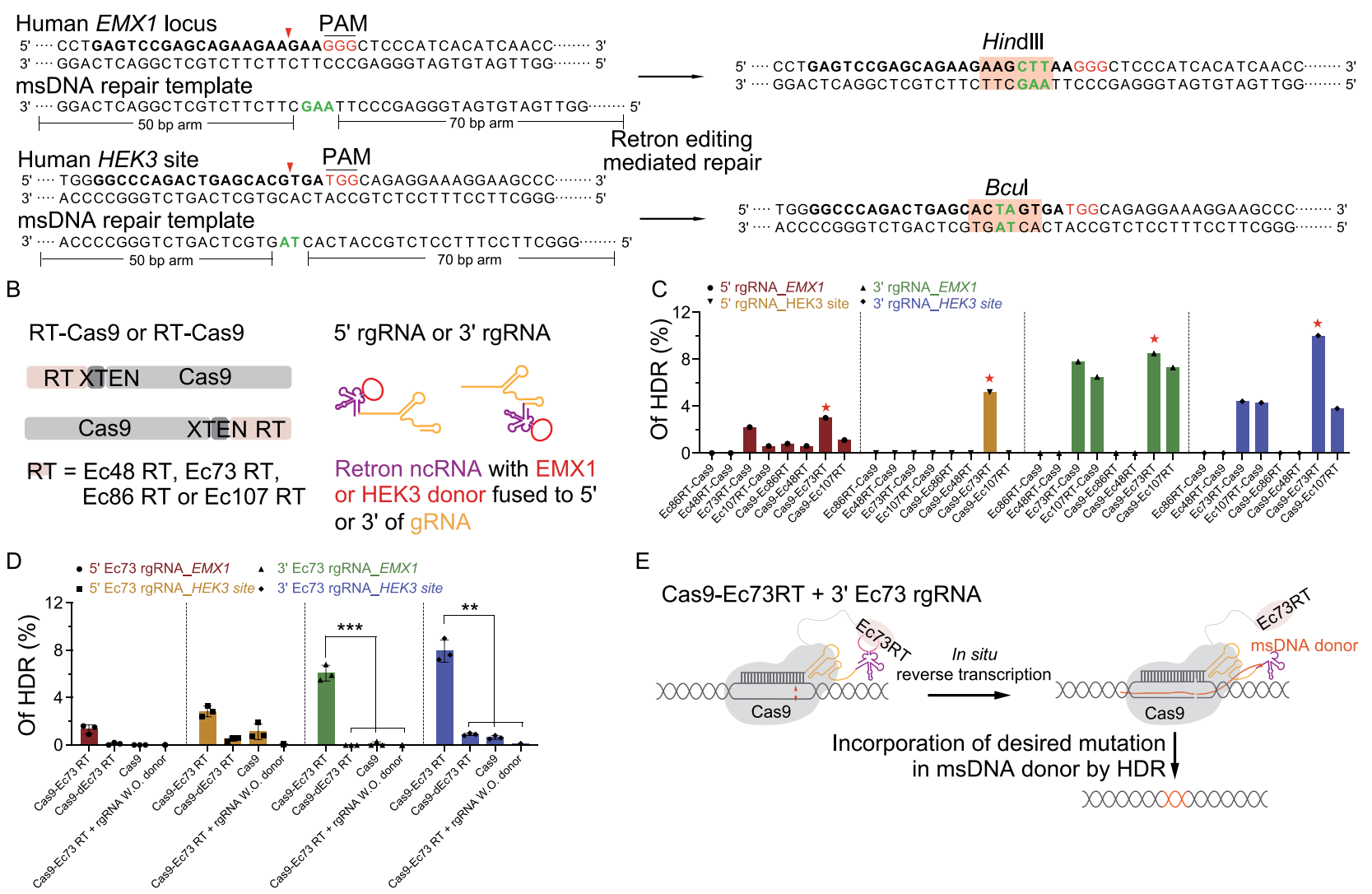

Figure 2. Retron editing system mediates precise editing at endogenous genomic loci. (A) Schematic of retron editing at human EMX1 locus and HEK3 site. The reverse-transcribed msDNA repair template complementary to the non-target strand is used as a repair template. The target sequence is in bold. The insertion sequence is marked in green letter. After retron editing, HindlII and $B c u l$ site is inserted into the EMX1 locus and HEK3 locus, respectively. (B) Plasmids construction strategies for retron editing system in human cells. Different Cas9-RT fusions targeting EMX1 or HEK3 combining with $5^{\prime}$ rgRNA or 3' rgRNA that can produce msDNA with homology donor sequences relative to EMX1 or HEK3, respectively, were co-expressed in HEK293T cells. (C) Analysis of retron editing efficiency by deep sequencing. (D) Deep sequencing to determine the efficiency of retron editing. dEc73 RT represents predicted catalytically dead Ec73 RT containing point mutation of D189A and D190A. rgRNA W.O. donor represents rgRNA without donor sequences inserted. ${ }^{* \star} P<0.001,{ }^{\star \star} P<0.01$, determined by Student's $t$-test, $n=3$. (E) Schematic diagram showing that Cas9Ec73 RT fusion in-situ reverse transcribe 3' Ec73 rgRNA to generate the msDNA containing a single-stranded donor sequence with homology arms, and msDNA is incorporated into the genome site cut by Cas9.

Additionally, retrons coupled with CRISPR can efficiently insert a GFP gene in yeast with efficiency up to $87 \%$ (Sharon et al., 2018), making us anticipating retron editing a more versatile genome editor in both therapeutic applications and crop improvement. Precise genome editing on locus corresponding to agronomic traits can greatly accelerate the crop breeding (Mao et al., 2019; Gao, 2021). Although, base transversions, small insertion and deletion by base editors and prime editors have been successfully achieved in plant cells (Gao, 2021). However, HDR-mediated large fragment insertions cannot be efficiently achieved due to the low HDR efficiency and limit of exogenous donor DNA delivery in plant cells (Gao, 2021). Therefore, it is interesting to test the possibility of using retron editing for large fragment insertion in plant cells.

Many approaches may be done to improve retron editing in human cells. Firstly, identification of more suitable retron for genome editing in human cells. Second, engineered evolution of both retron-RT and retron-ncRNA can be done to enhance retron mediated intracellular production of SsDNA. Finally, evidences were shown that hRAD51 mutant fused to Cas9 (D10A) nickase (RDN) fusions could mediate precise genome editing without DSBs (Rees et al., 2019). It is plausible to anticipate that combination of retrons with RDN may make retron editing more accurate and safer due to the elimination of Cas9-induced DSBs. 
To be noted, when we are preparing this manuscript, Zhao and colleagues preprinted their work of retron-mediated precise gene editing in human cells, which further convinced that retrons can be harnessed for precise genome editing in human cells by coupling with CRISPR-Cas9 (Zhao et al., 2021).

\section{FOOTNOTES}

This work was supported by HUIGENE Therapeutics Inc., the Basic Frontier Scientific Research Program of Chinese Academy of Sciences From 0 to 1 original innovation project (grant no. ZDBS-LYSM001), the R\&D Program of China (Grant Nos. 2017YFC1001300 and 2018YFC2000100) and the CAS Strategic Priority Research Program (Grant No. XDB32060000).

X.K. and H.Y. conceived and designed the project. X.K., Z.W., R. Z. and X.W. performed experiments and analyzed data. Y.Z. analyzed the data. X.K., L.S. and H.Y. wrote the manuscript.

All authors declare no conflict of interest. The authors declare that all other relevant data supporting the findings of this study is available within the paper and its Supplemental Materials, or from the corresponding authors upon request.

Xiangfeng Kong ${ }^{1 凶}$, Zikang Wang $^{2}$, Renxia Zhang ${ }^{2,3}$, Xing Wang ${ }^{1}$, Yingsi Zhou ${ }^{2}$, Linyu Shi ${ }^{1}$, Hui Yang ${ }^{2,4 \bowtie}$

${ }^{1}$ HUIGENE Therapeutics Inc., Shanghai 200131, China

2 Institute of Neuroscience, State Key Laboratory of Neuroscience, Key Laboratory of Primate Neurobiology, Center for Excellence in Brain Science and Intelligence Technology, Chinese Academy of Sciences, Shanghai 200031, China

${ }^{3}$ College of Life Sciences, University of Chinese Academy of Sciences, Beijing 100049, China

${ }^{4}$ Shanghai Center for Brain Science and Brain-Inspired Intelligence Technology, Shanghai 201210, China

$\bowtie$ Correspondence: xiangfengkong@huidagene.com (X. Kong), huiyang@ion.ac.cn (H. Yang)

Accepted June 26, 2021

\section{OPEN ACCESS}

This article is licensed under a Creative Commons Attribution 4.0 International License, which permits use, sharing, adaptation, distribution and reproduction in any medium or format, as long as you give appropriate credit to the original author(s) and the source, provide a link to the Creative Commons licence, and indicate if changes were made. The images or other third party material in this article are included in the article's Creative Commons licence, unless indicated otherwise in a credit line to the material. If material is not included in the article's Creative Commons licence and your

Xiangfeng Kong, Zikang Wang and Renxia Zhang have contributed equally to this work.

Supplementary Information The online version contains supplementary material available at https://doi.org/10.1007/s13238-02100862-7. intended use is not permitted by statutory regulation or exceeds the permitted use, you will need to obtain permission directly from the copyright holder. To view a copy of this licence, visit http:// creativecommons.org/licenses/by/4.0/.

\section{REFERENCES}

Anzalone AV, Randolph PB, Davis JR, Sousa AA, Koblan LW, Levy JM, Chen PJ, Wilson C, Newby GA, Raguram A et al (2019) Search-and-replace genome editing without double-strand breaks or donor DNA. Nature 576:149

Cox DB, Platt RJ, Zhang F (2015) Therapeutic genome editing: prospects and challenges. Nat Med 21:121-131

Dhundale A, Lampson B, Furuichi T, Inouye M, Inouye S (1987) Structure of msDNA from Myxococcus xanthus: evidence for a long, self-annealing RNA precursor for the covalently linked, branched RNA. Cell 51:1105-1112

Gao C (2021) Genome engineering for crop improvement and future agriculture. Cell 184:1621-1635

Hsu MY, Inouye S, Inouye M (1989) Structural requirements of the RNA precursor for the biosynthesis of the branched RNA-linked multicopy single-stranded DNA of Myxococcus xanthus. J Biol Chem 264:6214-6219

Lim H, Jun S, Park M, Lim J, Jeong J, Lee JH, Bang D (2020) Multiplex generation, tracking, and functional screening of substitution mutants using a CRISPR/retron system. ACS Synth Biol 9:1003-1009

Mao YF, Botella JR, Liu YG, Zhu JK (2019) Gene editing in plants: progress and challenges. Natl Sci Rev 6:421-437

Millman A, Bernheim A, Stokar-Avihail A, Fedorenko T, Voichek M, Leavitt A, Oppenheimer-Shaanan Y, Sorek R (2020) Bacterial retrons function in anti-phage defense. Cell 183:1551-1561

Mirochnitchenko O, Inouye S, Inouye M (1994) Production of singlestranded DNA in mammalian cells by means of a bacterial retron. J Biol Chem 269:2380-2383

Rees HA, Yeh WH, Liu DR (2019) Development of hRad51-Cas9 nickase fusions that mediate HDR without double-stranded breaks. Nat Commun 10:2212

Schubert MG, Goodman DB, Wannier TM, Kaur D, Farzadfard F, Lu TK, Shipman SL, Church GM (2021) High-throughput functional variant screens via in vivo production of single-stranded DNA. Proc Natl Acad Sci USA 118:18

Sharon E, Chen SA, Khosla NM, Smith JD, Pritchard JK, Fraser HB (2018) Functional genetic variants revealed by massively parallel precise genome editing. Cell 175:544-557

Simon AJ, Ellington AD, Finkelstein IJ (2019) Retrons and their applications in genome engineering. Nucleic Acids Res 47:11007-11019

Yee T, Furuichi T, Inouye S, Inouye M (1984) Multicopy singlestranded DNA isolated from a gram-negative bacterium, Myxococcus xanthus. Cell 38:203-209

Zhao B, Chen S-AA, Lee J, Fraser HB (2021) Bacterial retrons enable precise gene editing in human cells. BioRxiv. https://doi. org/10.1101/2021.03.29.437260 